\title{
Climate change, environmental impact, and human health
}

\author{
Bin Ye $\cdot$ Xiaolei Zhang $\cdot$ Xiaoling Zhang $\cdot$ Chunmiao Zheng
}

Published online: 7 April 2020

(C) Springer Nature B.V. 2020

Climate change is affecting ecological systems and human health in a profound manner. It contributes to the global burden of disease and leads to direct or indirect health impacts through extreme weather and climate events. According to IPCC report by many international scientist, global heating has caused serious and widespread impacts to human health. The challenges and problems we are facing are becoming systematic and inevitable.

Climate change is also regarded a critical driver of biodiversity loss and has a negative effect on food production, water resource availability, air quality, and ecosystem functions. In this context, it is important and valuable to examine perceptions from variegated stakeholders, including government, individuals, and other specialists to combat with the threat of climate change, their capacity to deal with it. We intend to develop and build an integrated

B. Ye $(\bowtie) \cdot$ C. Zheng

School of Environmental Science and Engineering,

Southern University of Science and Technology,

Shenzhen, China

e-mail: yeb@sustech.edu.cn

\section{Zhang}

Department of Civil and Environmental Engineering, Harbin Institute of Technology (Shenzhen), Shenzhen, China

X. Zhang

City University of Hong Kong, Hong Kong, China governance framework to assess the vulnerability, resilience as well as possibility of human health to climate change.

This special issue aims to present original research on climate change impact on environmental and human health, with a particular focus on the interaction of "Environment impact, Social cost and Human health." It consists of 21 peer-reviewed articles, which were originally selected from 48 articles submitted by researchers from around the world. These articles cover many topics related to climate change, environmental impact, and human health and focus on the following aspects:

1. For the ways climate change affects the environment, four papers studied water pollution caused by climate change and the ways climate change affect water quality and thus put forward corresponding countermeasures. Chen et al. used ultrasonication for the efficient removal of algae from water, which provides a low environmental impact process of water disinfection and thus reduces the adverse effect of climate change on water quality. $\mathrm{Fu}$ et al. studied the optimization of sulfur-based autotrophic denitrification by a highly enhanced denitrification process. The results help avoid the accumulation of $\mathrm{NO}_{2}-\mathrm{N}$ and improve the removal of nitrogen from wastewater. By using a trilateral evolution game model, Lu et al. proposed an evolutionary stable strategy to set up a cross- 
border water resource conflict warning system to address the upper, middle, and lower reaches in terms of water-quality conflict.

2. Mao et al. continued the water theme by studying the connections between human and natural ecosystems in an arid endorheic river basin where hydrological cycling was dramatically altered by human activities. Focusing on green and blue water in northwest China, they found that climate change and extensive water use reduce the availability of water for the natural ecosystem. Ye et al. found that combining $\mathrm{ClO}_{2}$ pre-oxidation and $\mathrm{ClO}_{2}$ disinfection provided an excellent water treatment reagent.

3. For the micro-ecosystem view, Dai et al. constructed the model aquatic micro-ecosystems to model the ecological effects of water diversion from Yangtze River to Lake Taihu on Gonghu Bay. The results revealed that the improvement in water diversion was positively correlated with the inflow discharges. Fei et al. studied the leaching behavior of arsenic from arsenic calcium residue (ACR) with different particle sizes in simulated acid rain with different acidities. The results indicated that smaller particles sizes and stronger acidity of leaching solution exhibited higher leaching concentration of arsenic.

4. For the impact of climate change on human health, two papers published by Liu et al. studied the impact of heavy metals on soil, which helps predict the risk of their presence in foods. They analyzed the spatiotemporal transformation and distribution of arsenic in the soil-crop system and pointed out the accumulation and distribution of heavy metal $\mathrm{Cd}$ in soils with reclaimed water drip irrigation in greenhouses during growing seasons. In another paper, Fan et al. found that the climate change observed in recent decades had impelled a significant shift in the pattern of traditional Chinese Medicinal Yam (CMY) planting areas, with global warming improving their climate suitability: The core CMY producing areas are mainly distributed in eastern Henan, southeastern Hebei, and western Shandong, China.

5. For the social impact perspective, $\mathrm{Lv}$ et al. introduced emergy theory as a new method to quantitatively study the relationship between environmental resources and social economy. It put forward a new evaluation method for using emergy loss as groundwater overexploitation ecological compensation. Fan et al. evaluated the impacts of climate change on residential energy consumption in different Chinese urban and rural regions. Shi et al. explored the long-term intervention effects of three types of policies (i.e., economic incentive policies) on resident's PM2.5 reduction intention.

6. For remedial measures, the development of emission trading schemes is examined by Zhou et al. in their systematic overview of seven emission trading scheme (ETS) pilots in China, involving the detailed comparison of ETS design and indepth evaluation of market performance, suggesting that a nationwide ETS should at least be based on an extension of the cap duration from single year to several years, uniform rules for monitoring/reporting/verification and allowance allocation, and improvement of the institutional foundation. Jiang et al. further pointed out that an effective and powerful regulation is indispensable for the development and smooth operation of a cap-and-trade ETS. These findings contribute to the establishment of China's nationwide ETS, and the development of ETS in other developing countries.

7. Another remedial measure is the development of policy, with two papers by Wang et al. exploring the effect of climate change information and emission policy on residents' health-risk perceptions and PM2.5 reduction behaviors. The results showed that while climate change necessarily has an adverse effect on human health, climate change information stimulates residents' enthusiasm for reducing emissions, thus contributing positively to human health. In contrast, Zhu et al. proposed a conceptual model that integrates the theory of planned behavior (TPB), smog knowledge, and risk perception, which aims to elucidate the determinants of smog-reduction behavior as a prelude to policy making. The result showed that knowledge of smog was a positive antecedent variable in risk perception, attitude, and perceived behavioral control. To trigger smog-reduction behavior, therefore, governments need to make efforts to spread information concerning the harmful effects of city smog.

8. Finally, it is the development of climate change monitoring and prediction models, with Sun et al. 
constructing three advanced mathematical grey prediction models to measure future national energy performance levels for both monitoring and reducing $\mathrm{NO}_{2}$ and other harmful GHGs emissions.

By presenting health assessment methods and econometric models as well as their applications and results, this special issue contributes to new progress in the exploration and discovery of the pathway, mechanism, and severity of climate change impact on human health. Climate change affects human health mainly through water, atmosphere, and soil. Thus, responsible stewardship of water, atmosphere, and soil will help to mitigate the adverse health effects of climate change.

All the submitted papers were subjected to the regular peer review process. We would like to thank all the authors and reviewers for their time and efforts involved.

Publisher's Note Springer Nature remains neutral with regard to jurisdictional claims in published maps and institutional affiliations. 